\title{
EL MODELO ECONÓMICO-POLÍTICO DE CHILE: DESARROLLO INSTITUCIONAL EN LA ENCRUCIJADA
}

\author{
Eduardo Saavedra P.*
}

\begin{abstract}
Resumen
Chile se caracteriza por un modelo económico-político que en las últimas tres décadas ha mostrado un importante desarrollo de sus instituciones más básicas, lo que le ha permitido obtener un fuerte crecimiento económico con progreso social y estabilidad política. Sin embargo, este mismo fortalecimiento institucional es la génesis de debilidades que le amenazan como proyecto, como son la exclusión social y la desigualdad de oportunidades. Para sustentar esta hipótesis, el trabajo se concentra en el desarrollo institucional chileno en cinco ámbitos: las reformas económicas, el desarrollo social, las instituciones económicas, las instituciones políticas y el consecuente clima de negocios que ha construido el país. La principal conclusión que emerge de este artículo es que, a pesar de ser considerado como un proyecto exitoso, este modelo económico-político chileno no ha logrado legitimarse del todo debido a que no ha sido capaz de garantizar certeza jurídica con inclusión social e igualdad de oportunidades.
\end{abstract}

Palabras clave: Institucionalidad, desarrollo, crecimiento, economía, política

* PhD en Economía. Profesor de Economía. ILADES-Universidad Alberto Hurtado. Correo electrónico: saavedra@uahurtado.cl.

El autor agradece los comentarios a una versión previa de este artículo de Eduardo Abarzúa, Claudio Agostini, Ilán Bizberg, Jorge Rodríguez y un árbitro anónimo. No obstante, las opiniones y errores que pueda contener este artículo son de su exclusiva responsabilidad. 


\title{
The Chilean Economic and Political Model: Institutional Development at the Crossroads
}

\begin{abstract}
Chile can be characterized as a country having an economic and political model that over the last three decades has shown an important development in its main institutions, which has allow to sustain strong economic growth together with social progress and political stability. However, the strengthening of its institutions is the origin of the main weaknesses that threat the country, i.e. the social exclusion and the increasing inequality of opportunities. To sustain this hypothesis, this work focuses on five areas of the Chilean institutional development: economic reforms, social development, economic institutions, political institutions, and the subsequent business climate the country has built. The main conclusion brought up by this article is that, despite being considered as a successful project, the economic and political Chilean model has not reach complete legitimacy because it has not been able to guarantee legal certainty with social inclusion and equality of opportunities.
\end{abstract}

Keywords: Institutions, development, growth, economics, politics 


\section{Introducción}

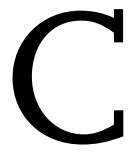

hile es un pequeño laboratorio en donde se han implementado diversos modelos económicos y políticos que, de una forma $u$ otra, han sido objeto de réplica en otros países de América Latina. Pasando por la experiencia socialista de la Unidad Popular (I970-1973), siguiendo por el gobierno militar y el apoyo de los sectores neoliberales (I973-I990), se ha llegado a un modelo económico-político que mezcla esos dos mundos que le precedieron.

Este artículo desentraña cuál es el modelo económico-político del Chile actual. La hipótesis central es que una de las principales características de este modelo es la fortaleza de sus instituciones más básicas, tanto económicas como políticas, que le han permitido mostrar como país un mayor nivel de desarrollo respecto al de su vecindario. Con todo, se han manifestado recientemente en Chile ciertas falencias inherentes a este mismo desarrollo institucional. Este trabajo se inscribe así en la literatura que enfatiza la idea de que el desarrollo de las instituciones es la base para el desarrollo de los países. ${ }^{\mathrm{I}}$

El modelo económico-político chileno es el de una economía de mercado con fuerte énfasis social. Esto es, protege fuertemente la libertad de emprendimiento y los derechos de propiedad, asignando al Estado un rol subsidiario y no empresarial. Conforme a ello, el Estado en Chile tiene un papel regulador en tanto corrector de fallas de mercado (poder de mercado y existencia de mercados incompletos) y fallas sociales (pobreza,

${ }^{1}$ La importancia de las instituciones en el crecimiento económico no está en duda, como bien lo plantea North (I990), revisa Keefer (2004), y más recientemente ha hecho popular Acemoglu y Robinson (20I2). La causalidad entre desarrollo institucional y crecimiento económico aparentemente se debería a la aparición de una serie de instituciones 'híbridas' en economías de mercado que son cruciales para generar las condiciones para el crecimiento económico (Menard 2006). 
seguridad social, desigualdad). En consecuencia, Chile muestra un desarrollo institucional cuyo norte económico es entregar certeza jurídica y equidad de trato al mundo empresarial, pero dando importancia, a modo de contrabalance de este liberalismo, a un Estado protector del bien común. En palabras de Schneider y Soskice (2009), nuestro sistema económico-político es de tipo 'liberal', a diferencia de otros modelos capitalistas que los autores llaman 'coordinados' y 'jerárquicos'.

Para entender esta economía social de mercado es bueno poner como contrafactuales los modelos polares de desarrollo que el propio Chile ha mostrado en los últimos 40 años: el modelo socialista de Salvador Allende y el neoliberal de Augusto Pinochet. El proyecto de Allende en Chile, y de alguna manera los más recientes en Venezuela, Bolivia y Ecuador, puede ser caracterizado como un sistema planificador, de alta participación social y con un Estado empresarial muy fuerte, y en el cual el mercado tenía un rol minimizado: controles de precios generalizados; control de capitales, tanto interno como externo; control al comercio internacional y fuerte proteccionismo con el fin de fomentar el desarrollo de la industria doméstica. Su principal característica fue, sin duda, el fuerte énfasis en lo social.

El otro proyecto polar fue el neoliberal, que en Chile tuvo su manifestación en dictadura. Este modelo se caracteriza por una liberalización 'extrema' de muchos mercados, aun aquellos con claras fallas como el de servicios básicos, en donde algunos segmentos de los mercados de electricidad y agua estaban completamente liberalizados; los de necesidad básica, en donde se avanzó fuertemente en la privatización de la educación y la salud; o el del transporte público totalmente desregulado. Consistentemente, este modelo se caracteriza por un débil rol regulador y un aún más bajo énfasis en la política social.

El modelo económico-político chileno actual se relaciona, aunque no con exclusividad, a la Concertación de Partidos por la Democracia (Concertación, de acá en adelante), en el poder desde I990 a 20ı0. Esta es una coalición compuesta por los partidos políticos que, siendo contrarios a la dictadura, se mostraron proclives a mantener y corregir, en lugar de cambiar, el modelo neoliberal heredado. ${ }^{2}$

\footnotetext{
${ }^{2}$ Con matices menores, el gobierno de Sebastián Piñera (2010-20I4), apoyado por la Alianza por Chile (Alianza, de acá en adelante), coalición mayoritariamente ligada a la conducción económica y política de la dictadura, prosiguió con el mismo modelo económico-político, sin mostrarse más liberal ni menos social que sus predecesores de la Concertación. Con todo, no es posible aseverar si este camino se optó por convicción o simplemente por la imposibilidad de cambiar la institucionalidad ya construida en los veinte años previos.
} 
La Concertación ha vuelto al poder en 2014, pero en un frente más amplio (Nueva Mayoría) que incluye al Partido Comunista y en donde los detractores del modelo han hecho ver con más fuerzas su deseo de avanzar hacia un 'modelo de desarrollo' alternativo. Estos detractores del modelo actual plantean la necesidad de generar mayor inclusión social y atacar la desigualdad, aunque ello afectase el desarrollo institucional que el país ha construido en las últimas dos décadas. Sin saber exactamente en qué consiste esta suerte de modelo alternativo, se pueden identificar algunos ámbitos de controversia dentro de la misma coalición de partidos que son relevantes de precisar.

Si nos circunscribimos sólo a aspectos de economía pública, se puede encontrar algún grado de debate en torno a los siguientes fenómenos. En temas de institucionalidad regulatoria: el dilema de hasta dónde la certeza jurídica debe ser un objetivo que amenace incluso el principio de más y mejor competencia o el de protección de los consumidores. En materia laboral: flexibilidad laboral versus la protección de los derechos del trabajador; o el cuestionamiento al sistema de previsión social. En aspectos de política social: sólo reducción de la pobreza o avanzar en la inclusión social y la reducción de la desigualdad. Por último, en los servicios de necesidad básica: si el rol del Estado debe ser garantizar la calidad y acceso equitativo o sólo perseguir estándares mínimos garantizados, cuestionándose incluso el rol subsidiario del Estado en estos servicios.

La sección siguiente muestra los elementos que caracterizan al modelo económico-político chileno. La tercera sección se adentra en las reformas económicas y sociales que ha experimentado Chile desde los años I970 y que han estructurado su desarrollo económico reciente. La cuarta sección muestra los principales aspectos de la institucionalidad económica y la institucionalidad política chilena. Más que como un fundamento del modelo chileno, los indicadores del clima de negocios en el país que se muestran en la sección cinco son el resultado de las políticas implementadas. A modo de conclusión, la sección seis muestra algunas propuestas que modifican la institucionalidad económica y entregan al modelo actual elementos de mayor inclusión social desde adentro. 


\section{Características del modelo económico-político chileno}

En marzo de 1981 entró en vigencia la nueva Constitución Política de la República de Chile. Esta vino a reemplazar a la Constitución de I925, la que, a su vez, había reemplazado a la de I833. Es fácil ver la estabilidad de la carta fundamental que muestra Chile como país. A juicio de sus precursores, la Constitución de I980 se hacía necesaria en tanto su antecesora mostraba deficiencias que se habían acentuado en los últimos años, las que habrían posibilitado con consecuencias lógicas y predecibles el quiebre democrático que devino en el golpe militar de I973. Más allá de un juicio a dichas aseveraciones, esta nueva Constitución significó un cambio profundo en la concepción política y económica del país, sentando las bases definitivas de lo que podemos llamar el modelo económico-político de Chile.

La Constitución de I980 es ciertamente conservadora en lo valórico, poco democrática (de forma y fondo) y con un fuerte sesgo económico liberal. A modo de ejemplo, pero muy clarificador, es que en ella se protege expresamente el derecho de propiedad, mientras que no se hace mención a otros derechos fundamentales, como los derechos humanos, por ejemplo. La consecuencia económica de esta Constitución, y de sus diversas leyes, es el haber dotado de certeza jurídica a las inversiones privadas, creando así un adecuado clima de negocios. Esto a su vez significó una estabilidad económica sin precedentes en la historia de Chile, con más de dos décadas de crecimiento económico sostenido que han cambiado la faz del país. Con la llegada de la democracia en I990, los gobiernos de la Concertación abrazaron este modelo liberal, muchos por omisión más que por convicción, y lo dotaron de un matiz social con modificaciones legales construyendo así un proyecto con discriminación positiva hacia los más pobres. Esto es lo que el Presidente Aylwin (I990I994) llamó 'crecimiento con equidad'. Como veremos, más allá del eslogan, se han implementado programas sociales tendientes a reducir la pobreza, pero permanece pendiente la alarmante carencia en aspectos distributivos. ${ }^{3}$

${ }_{3}^{3}$ Una muy buena referencia es Foxley (I993). Una mirada crítica a este paradigma, aunque aún dentro de la Concertación, la entregan Meller (2005) y Raczynki y Serrano (2005). 
El actual modelo chileno tiene la carga del modelo original, especialmente en lo económico, pues se caracteriza por una serie de políticas económicas como la responsabilidad fiscal, la competitividad del país, la estabilidad en las reglas del juego, el garantizar los derechos de propiedad, la creación de instituciones sólidas y bien diseñadas, la continuidad y estabilidad política y la paz social. Sin embargo, el modelo de economía social de mercado surgió como respuesta a la preocupación de los gobiernos democráticos de encontrar el adecuado soporte político para garantizar la continuidad política y la responsabilidad fiscal. Así, se construyó un consenso político acerca de la estrategia de desarrollo nacional. No obstante, no todo es tan positivo, ya que aquellos que discrepaban del modelo, por muy menores que hayan sido, quedaron excluidos de toda posibilidad de participar en él. En otras palabras, su propia fortaleza (estabilidad en las reglas del juego) devino en su propia debilidad (exclusión social).

La Figura I esquematiza las cinco claves del modelo económicopolítico del Chile actual: las reformas económicas, su desarrollo social, las instituciones económicas, las instituciones políticas y el consecuente clima de negocios que surge.

- Figura 1. Aspectos CLAVE del modelo chileno

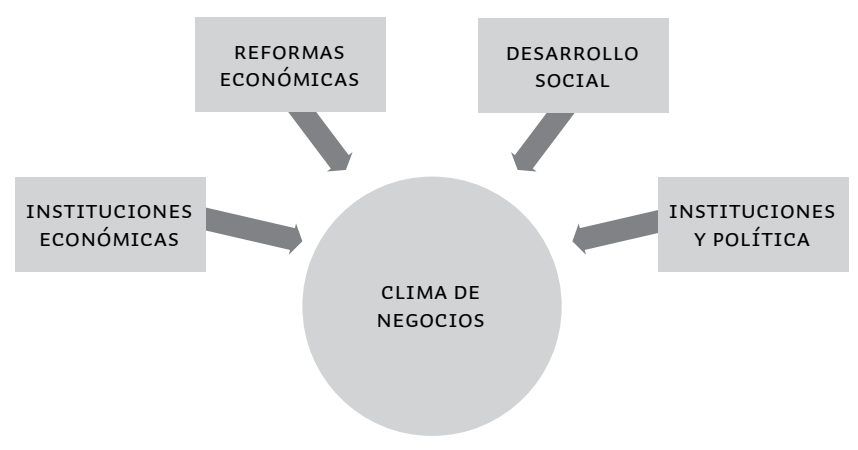




\section{Reformas económicas y progreso social}

Las primeras reformas económicas que dieron cuerpo al modelo chileno surgieron como respuesta al modelo planificador de Allende apenas ocurrido el golpe militar de I973. Las reformas en materia fiscal, comercial, de capitales y financiera, y laboral acometidas en los años I970 fueron consistentemente modificadas en los años I980 para viabilizar el modelo neoliberal. ${ }^{4}$

La diferencia más fuerte con la historia económica chilena del siglo XX descansa en que el modelo neoliberal controló de raíz el crónico problema inflacionario, por la vía de controlar el déficit fiscal y la emisión de dinero. Una serie de reformas al gasto público, que devinieron en un brutal desempleo en 1975 y años posteriores, cortaron el déficit fiscal desde un 24,7\% del PIB en I973 a un 2,6\% en I975. La caída en el gasto público fue implementada por la vía de reducir el empleo público y controlar las pérdidas en las empresas estatales, principalmente a través de reprivatizaciones de empresas estatizadas durante el gobierno de Allende. La reforma tributaria fue otro hito en los años I970: se modificó el sistema de impuesto al consumo en cascada por el impuesto al valor agregado en 1975 .

Podría parecer que las reformas fiscales fueron sólo de tenor macroeconómico neoliberal y tendientes a controlar la inflación. No obstante, le siguieron otras reformas en estos ámbitos y todas mostraron el compromiso institucional hacia el desarrollo de una economía de mercado, las que fueron hechas tanto en tiempos de dictadura como en democracia. En el ámbito del gasto fiscal es interesante el compromiso de superávit fiscal impuesto hacia fines del gobierno de Eduardo Frei Ruiz-Tagle (I994-2000), el que consiste en el compromiso del gobierno de mantener un superávit fiscal equivalente al ı\% del PIB potencial, cifra modificada a la baja posteriormente en el gobierno de Michelle Bachelet (2006-2010).

En el ámbito tributario, ya en los años I980 se modificó la estructura de impuestos a las empresas y a las personas, las que, si bien siempre buscaron favorecer la reinversión de utilidades de las empresas

${ }^{4}$ La discusión que sigue se basa en los trabajos de Edwards (200I), Foxley (2005), Landerretche (2005) y Larraín y Vergara (200I). Una mirada crítica de este proceso de desarrollo en Chile se puede encontrar en Ffrench-Davis (200I). 
por la vía de imponer una tasa de impuesto única a las empresas de un I5\%, significaron una política regresiva por cuanto han sido las personas de más recursos las que han podido utilizar franquicias a la hora de declarar impuestos, estrategia que no es posible para la clase media que tributa en base a sus ingresos, en la mayoría de los casos como trabajadores dependientes. Posteriores modificaciones a esta estructura de impuestos han sido más bien cosméticas (como el alza y baja del IVA que ha ido entre el I $8 \%$ y el I $9 \%$, impuestos a las empresas que se subieron al I7\% y luego al 20\%, y una serie de nuevas exenciones a las inversiones de largo plazo).

Las privatizaciones de empresas en el gobierno militar mostraron que éstas no sólo eran fruto de las necesidades de caja del sector público, sino principalmente ideológicas. A las privatizaciones de empresas en sectores productivos en los años I970 le siguieron privatizaciones en mercados con características monopólicas en los I980, como en las telecomunicaciones en I987 y en el sector eléctrico en I990. Ahora bien, quizás menos por ideología que por necesidad de nuevas inversiones, fue en la época democrática en que se continuó con estas privatizaciones alcanzando al siempre sensible sector de empresas sanitarias (agua potable y alcantarillado).

Por último, respecto del tema del gasto público, el Estado ha ido reduciendo su importancia en Chile incluso a través de nuevas iniciativas que surgieron en la era de los gobiernos de la Concertación. Fue así como en I993 se inauguró el proceso de concesiones de obras de infraestructura, una forma indirecta de atraer capital privado a sectores típicamente reservados sólo para la inversión pública, como carreteras urbanas e interurbanas, puertos y aeropuertos, cárceles y, últimamente, hospitales, entre otros.

Otra política consistente con el modelo económico imperante fue la apertura de Chile hacia el resto del mundo, tanto por el lado de la política comercial como por el de capitales. Previo al golpe militar, la tasa arancelaria promedio de Chile superaba el Ioo\%, con una enorme varianza al observarse, por un lado, exenciones arancelarias y, por otro, tasas a ciertos productos que superaban incluso el Iо००\%. Ante aquel panorama se optó por homogeneizar las tasas arancelarias a niveles que fueron paulatinamente bajando hasta el I5\% en I989. La política seguida 
por Chile en dicho período fue la rebaja unilateral en los aranceles como una manera de abrirse al mundo, abandonando además los acuerdos comerciales sostenidos con países vecinos, como el Pacto Andino (que se abandonó en I977).

Desde I990, los gobiernos de la Concertación siguieron con una política de bajas arancelarias pero con un énfasis en acuerdos de libre comercio firmados con sus principales socios comerciales: Estados Unidos, Japón, la Unión Europea y China. Actualmente la tasa arancelaria nominal de Chile es del 7\%; empero, producto de acuerdos comerciales que mantiene con más de cincuenta países, ésta llega en promedio al I\%.

Los flujos de capitales en Chile fueron totalmente liberalizados hacia fines de los años I970. Sin embargo, el colapso económico de I98283 cerró nuevamente el flujo de capitales. En los años I990 el Banco Central de Chile mantuvo una política de encajes que desincentivaba la entrada de capitales de corto plazo. Finalmente, hacia fines de los años I990, y luego de casi dos décadas desde la debacle de I982-83, Chile abrió nuevamente la cuenta de capitales y los flujos desde y hacia el exterior sin mayores restricciones.

Dentro del mercado de capitales doméstico, varias sonlas reformas que se hicieron a lo largo de estas últimas tres décadas. Sin dudas, y por lejos, la reforma más importante en magnitud, en influencia sobre el resto de la economía y en lo novedoso de la iniciativa, fue la introducción de un sistema de capitalización privada en la seguridad social. El efecto más importante de esta reforma fue permitir la acumulación de recursos financieros significativos para ser invertidos en el mercado de capitales. El sistema de fondos de pensiones ha acumulado al año 2012 recursos cercanos al 70\% del PIB (Fuentes 20I3); esto sin contar lo acumulado por las compañías de seguros de vida, industria que basa su desarrollo fuertemente en los cambios en el sistema previsional.

En I980, el gobierno militar modificó el antiguo sistema de seguridad social de reparto o pay as you go por uno de capitalización individual o fully-funded system. Esta modificación planteó además un cambio mayor en el desarrollo del mercado de capitales del país. Es así como en I98I se dictó la ley orgánica que creó la Superintendencia de Valores y Seguros, la ley de mercado de valores y la ley de sociedades anónimas, cuyos objetivos fundamentales fue establecer un marco 
jurídico armónico que permitiera el desarrollo de una intermediación de valores de oferta pública con participación de inversionistas institucionales.

Con posterioridad a la crisis de $1982-83$ se introdujeron diversas reformas a la regulación de los inversionistas institucionales y al mercado de valores con el fin de fortalecer el sistema privado de pensiones; un reflejo de esto es que sólo en I986 se permitió la inversión en instrumentos de riesgo. De igual modo, la reforma al mercado de capitales de 1993 fue la constatación de que la escasez de opciones de inversión para los fondos de pensiones implicaba una peligrosa concentración de los portafolios, afectando la rentabilidad y aumentando el riesgo de los fondos. Igualmente, a fines de los I990 se permitió a las AFPs aumentar su inversión en activos financieros externos, lo que ayudó en gran parte a remediar el problema de falta de diversificación de los fondos de pensiones.

La reforma más profunda al sistema de pensiones en Chile se implementó en 2008, durante el primer gobierno de Michelle Bachelet. El énfasis principal de esta reforma fue el fortalecimiento del pilar solidario del sistema de pensiones, ampliando los beneficios de pensión mínima (renombrada como aporte previsional solidario) y de pensión asistencial (renombrada como pensión básica solidaria). Asimismo, se hizo obligatorio el ahorro de los trabajadores independientes, se reguló de mejor forma el conflicto de interés de las AFPs respecto del seguro de invalidez y sobrevivencia y se introdujo la licitación de la cartera de nuevos afiliados al sistema.

En paralelo, pero fuertemente influido por la reforma a la seguridad social, Chile ha experimentado sucesivas reformas al mercado financiero. Estas reformas apuntaron a liberalizar el sistema en forma consistente con lo ocurrido con los capitales externos. Sin embargo, la crisis económica de principios de la década de los I980 llevó a una re-regulación del sistema bancario de tal magnitud que incluso en la actualidad diversas operaciones bancarias, como los préstamos relacionados o los préstamos cruzados o triangulares, están regulados y a veces incluso prohibidos. 
De hecho, la estructura de los bancos comerciales está fuertemente regulada para evitar la propagación del riesgo sistémico, llegando al punto de que los bancos no pueden poseer acciones de empresas productivas (se separaron los bancos comerciales de los bancos de inversión) y, hasta principios de la década pasada, los negocios relacionados a actividades bancarias fueron permitidos sólo si eran operados por filiales bancarias.

Respecto de las reformas laborales, Chile es un país en extremo desregulado, contrario a lo que sucede en el mercado de capitales. Hacia fines de la década de i970 se creó un nuevo código laboral que normó, entre otras cosas, la libertad de contratación y despido de trabajadores, y el alcance de los sindicatos y rol de las negociaciones salariales en el sector privado. Este código laboral en lo medular autoriza la libre contratación de los trabajadores, el cumplimiento de las leyes sociales en cuanto al aporte a la seguridad social y a los seguros de salud, y las indemnizaciones en caso de despido y las causales de despido.

En forma consistente con esta mayor desprotección de los trabajadores, el código laboral redujo a su mínima expresión el rol de los sindicatos y debilitó fuertemente la posición negociadora de los trabajadores, respecto de la situación precedente. En efecto, basta notar que se prohibió la negociación por rama de actividad económica y se normó el proceso de negociación colectiva y se restringió el derecho a huelga; pero, por sobre todo, se permitió a las empresas contratar trabajadores de reemplazo en caso de que sus empleados estén en huelga legal. Esto, como veremos, ha tenido serias consecuencias en el crecimiento económico (le favorece) y en la protección social (le desfavorece).

La llegada de la democracia modificó sólo cosméticamente esta relación trabajador-empleador. Por ejemplo, se modificó el que no hubiese causal de despido por un despido 'por necesidad de la empresa'. A su vez, los intentos de modificaciones tendientes a mejorar la posición de los trabajadores han sido sistemáticamente anulados por el poder legislativo.

El modelo neoliberal implementado en Chile durante la dictadura militar no mostró especial cuidado en las consecuencias sociales de las políticas económicas del régimen. No obstante, algunas medidas que se 
tomaron en esos años se focalizaron en el gasto social hacia los sectores más vulnerables. Con todo, el gasto social en los años I980 tuvo el valor más bajo de la historia económica reciente de Chile, generando importantes déficits en atención de salud, apoyo a los grupos más vulnerables, vivienda y el gasto en educación, particularmente primaria.

Los resultados en el desempeño se han visto influidos por las reformas económicas implementadas, y en los matices entre los diversos gobiernos. Desde I990 a 2012 el producto interno bruto per cápita creció en I29\%, según se muestra en el Gráfico I.

- GRÁFICO 1. PIB PER CáPITA EN CHILE Y AMÉRICA LATINA

(eN DólARES A PRECIOS CONSTANTES DE 2005)

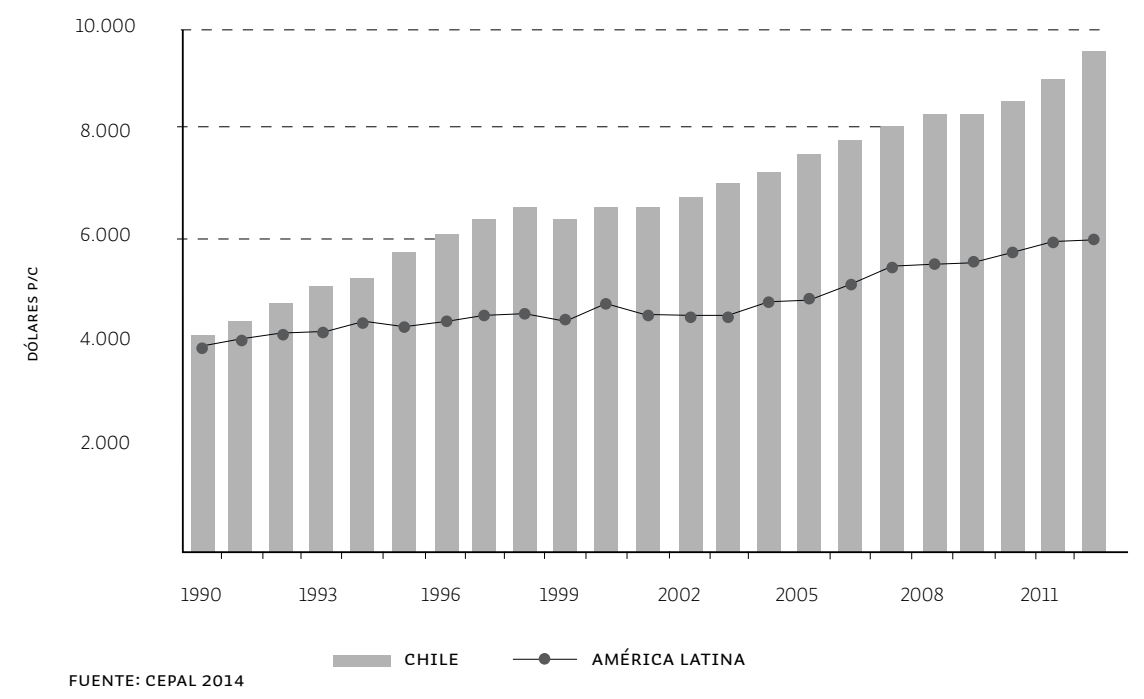

Como comparación, se observa que América Latina vio crecer este indicador en el mismo período en menos de un $48 \%$ (todo en dólares corrientes y no corregido por paridad en el poder de adquisición). Si se observa la tasa de crecimiento anual del PIB en Chile (Gráfico 2), ésta muestra dos grandes períodos. El primero de ellos ocurre con la recuperación de la recesión de los años I982 y i983. La llegada de la democracia y sus readecuaciones sociales no redujeron el ritmo de crecimiento sino que lo mantuvo, a pesar de que para i990 la tasa 
de desempleo ya había llegado a su nivel de pleno empleo. En otras palabras, el crecimiento de toda la década de los I990 fue exclusivamente crecimiento neto.

- GRÁfico 2. TASA DE CRECIMIENTO ANUAL DEL PIB EN CHILE

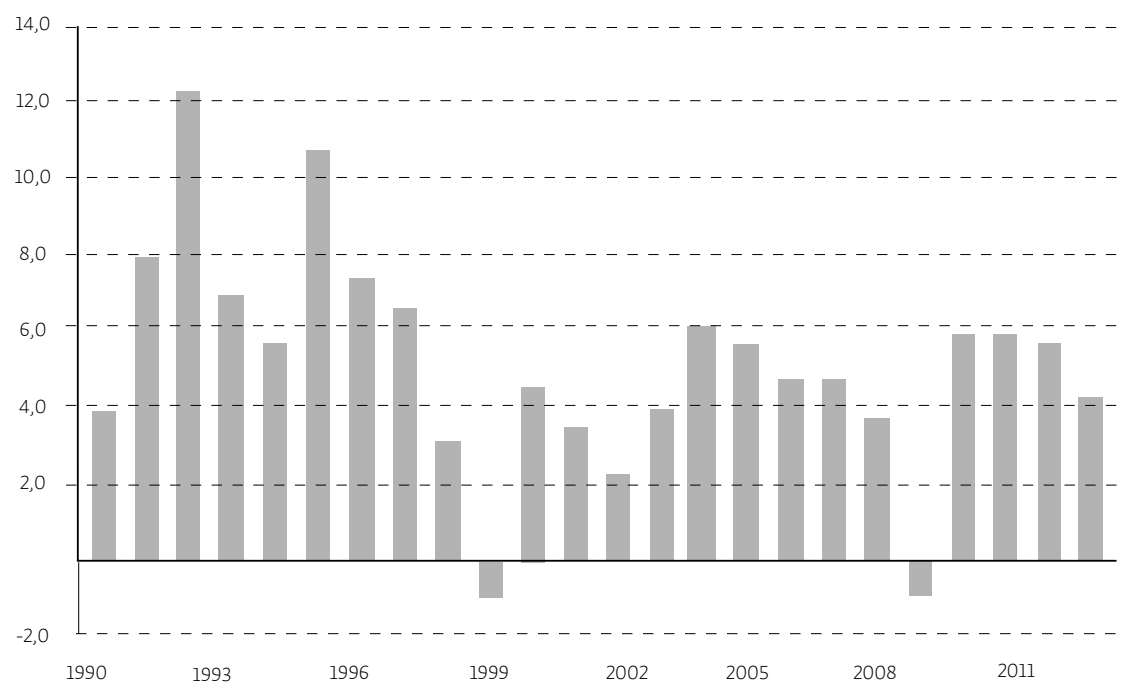

FUENTE: CEPAL 2014

Se observa en la serie una desaceleración de la actividad económica posterior a la crisis asiática de I998. La tasa de crecimiento económico de los años previos, que alcanzó en promedio a casi un $8 \%$ anual -lo que, descontado el crecimiento vegetativo de la población en torno a un I\%, da tasas de crecimiento per cápita cercanas al 7\%- no volvió a repetirse. Así, la tasa de crecimiento anual del PIB desde el año 2000 alcanzó un 4,2\%; es decir, un 3,2\% per cápita por año. En términos comparativos, con la tasa de los años 1985 a I998 el país duplicó su ingreso per cápita cada io años, mientras que al ritmo posterior a la crisis asiática -que incluye la crisis de la deuda subprime que golpeó a Chile en 2009- sólo lo puede hacer en 23 años.

La política social focalizada y sobre todo incrementada en la década de 1990 en adelante, junto por supuesto al significativo crecimiento económico de Chile en los últimos 25 años, bajó la tasa de pobreza desde 
un $38,4 \%$ en I990 a un I4,4\% en 20 II. Igualmente, la tasa de indigencia, o de extrema pobreza, se redujo considerablemente desde un I $2,8 \%$ a un 2,8\% en el mismo período, según se muestra en el Gráfico 3.

- GRÁFICO 3. POBREZA E INDIGENCIA EN CHILE

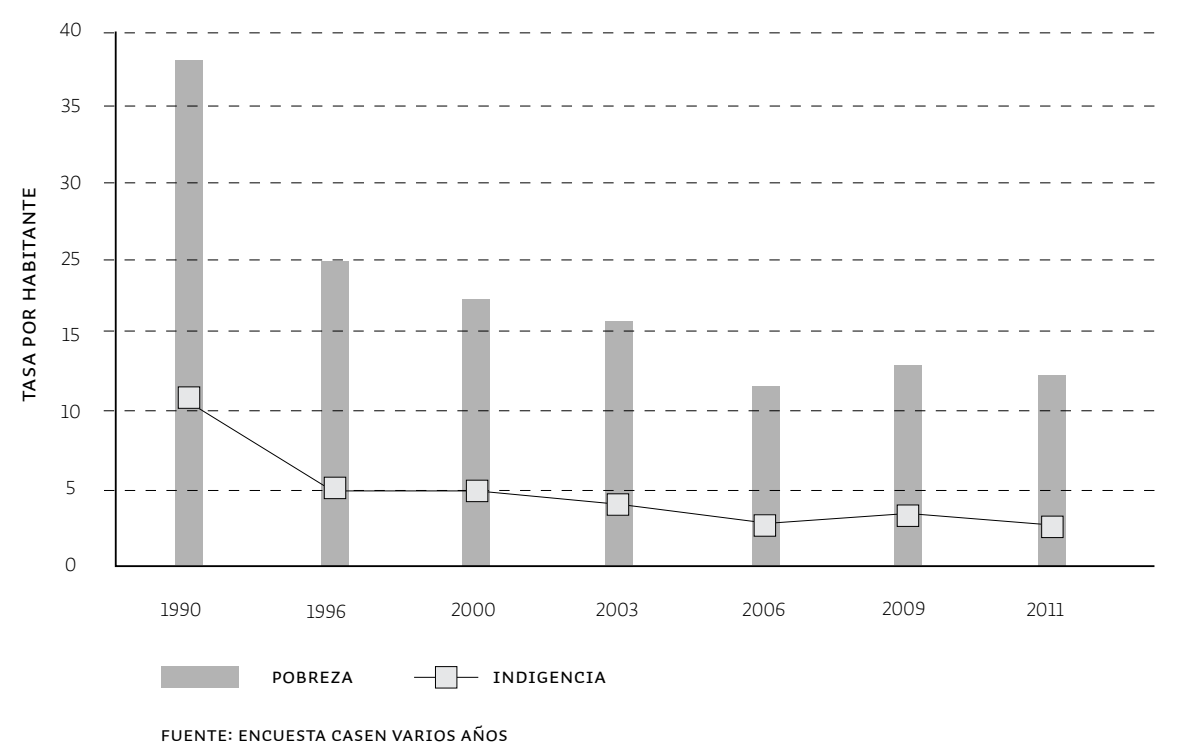

Probablemente la consecuencia más importante del sostenido crecimiento económico chileno sea la reducción de la pobreza, junto con el aumento en la calidad de vida del promedio de la población. Sin embargo, ni el modelo de mercado ni la política social tendiente a aliviar sus consecuencias adversas han logrado resolver los problemas de desigualdad. A modo de ejemplo, la desigualdad en los ingresos medida a través del Coeficiente de Gini ha caído sólo un $7 \%$ en los últimos veinte años. Igual situación de baja respuesta en los indicadores de desigualdad a la política social se aprecia utilizando los ratios del quinto a primer quintil y del décimo a primer decil, según se muestra en el Gráfico 4. Es decir, a pesar del crecimiento y la reducción en la pobreza, Chile es casi tan desigual como lo era antes de esos evidentes logros económicos. 
- GRÁFICO 4. DESIGUALDAD DE INGRESOS EN CHILE

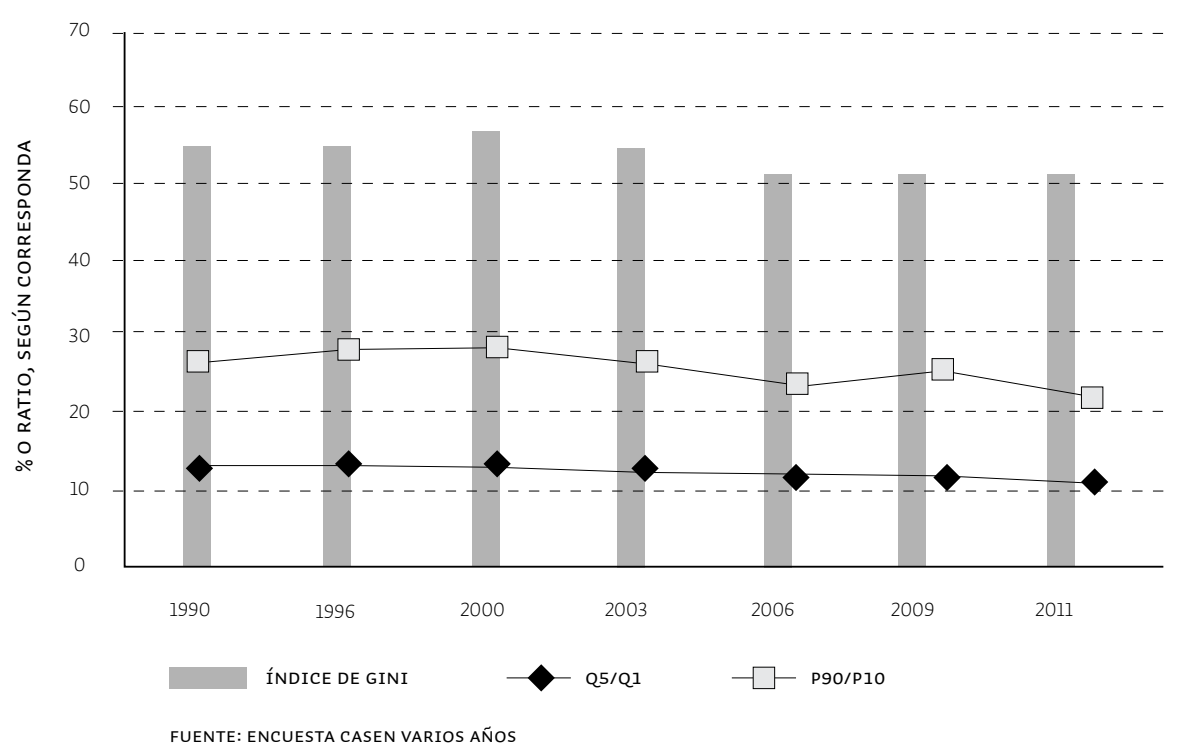

Subyacente a esta pobre distribución de los ingresos están los problemas de desigualdad en el acceso a educación, salud, vivienda y protección social de la población. A pesar del aumento en el gasto social supuestamente focalizado a los más pobres, cuando se consideran las transferencias directas del fisco el Coeficiente de Gini llega a sólo un $52 \%$ en 20II. Es decir, a pesar de toda la ayuda y su creciente mayor riqueza relativa, Chile sigue siendo más desigual que el promedio de América Latina, sólo siendo superado por Brasil, Colombia, Guatemala, Honduras, Panamá y Paraguay.

Los indicadores sociales tampoco muestran avances sustantivos, lo que es consistente con la historia de inequidad en el acceso. Esto a pesar de que, si se miran sin mucho análisis algunos indicadores, Chile aparecería mejor en la tasa de analfabetismo (bajó levemente desde un I,6\% a un I,I\% entre I992 y 2009 para la población entre I5 y 24 años), en la escolaridad promedio de la población pobre (el primer quintil aumentó para la población activa desde 7,3 años en I990 a 9,I años en 20II), y en el acceso a la salud, vivienda y protección social (indicadores muestran una tendencia hacia la mejoría). 
Con todo, si se observan en más detalle esos indicadores es posible detectar brechas crecientes en el acceso y calidad de la educación. Por ejemplo, si bien los años de escolaridad de los más pobres han aumentado, la calidad de ésta sigue estando muy por debajo de la que tienen los grupos más privilegiados. Esto es, los pobres van más tiempo a las aulas, pero guarismos como el indicado no reflejan que la brecha en el acceso a bienes de necesidad básica sea creciente; es decir, los pobres tienen acceso a más tiempo de escolaridad, pero no a una mejor calidad en la educación. Iguales patrones se observan en la mirada más detallada de indicadores en áreas como la salud o la vivienda.

Esta alta y persistente desigualdad, junto a las pocas oportunidades para la movilidad social -en particular para acceder a los deciles de mayor ingreso-, genera una baja cohesión social. De acuerdo a información de CEPAL, Chile muestra estadísticas que están levemente mejor que el promedio de América Latina en términos de cohesión social, pero en prácticamente todos los indicadores está por debajo de Argentina y Uruguay, los otros dos países del Cono Sur.

A modo de resumen, siguiendo a Bourguignon (2003) es posible categorizar la relación entre crecimiento, pobreza y desigualdad en Chile. La Figura 2, tomada de Rodríguez y Saavedra (2008), nos muestra que Chile lo ha hecho muy bien en términos de crecimiento económico, siendo el ideal un crecimiento anual de $7,5 \%$ (equivalente al promedio del mayor crecimiento de un país de América Latina año tras año entre I990 y 2006), y Chile está en el 74\% de ese valor. Igual situación se da en el indicador de pobreza, siendo el ideal que ésta llegara a un $8 \%$ (promedio de los países de la OECD), estando Chile en un $84 \%$ de ese ideal. Sin embargo, la situación de la desigualdad de los ingresos está estancada. De más está decir que esta situación no dista mucho de la observada para prácticamente toda América Latina, aunque las diferencias entre los diversos modelos seguidos va más allá de simples matices de capitalismo (Bizberg 20I4). 


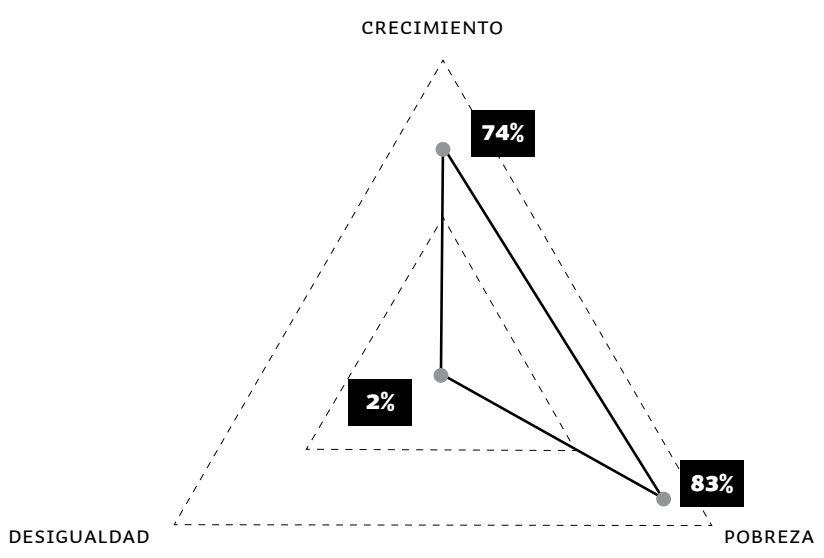

FUENTE: RODRÍGUEZ Y SAAVEDRA 2008

\section{Instituciones económicas y políticas en Chile}

Chile ha ido construyendo y fortaleciendo sus instituciones desde la época colonial. Ya como República, la Constitución de I833 entregó una fuerte institucionalidad y una consecuente estabilidad al país. Igual logro tuvo la Constitución de i925. En consecuencia, la fortaleza institucional que muestra Chile es consecuencia de una larga evolución y no puede necesariamente asignársele sólo a su pasado más reciente. ${ }^{5}$

La Constitución de I980 vino a refrendar esta institucionalidad vigente en sus principales aspectos, pero además imprimió un fuerte sesgo económico (y liberal) a la Carta Magna que, como hemos visto, ha influido fuertemente en el devenir de los últimos 30 años. Dos son las principales características de la institucionalidad política que me interesa destacar: el sistema electoral y los mecanismos de checks and balances.

El sistema electoral binominal que tenemos en Chile se aplica para la elección de senadores y diputados. En cada caso, la primera mayoría es inmediatamente elegida, y el segundo electo será de la coalición que obtenga la primera mayoría sólo en caso que doble en

5 Esta sección se basa libremente en varios autores, entre los que destaco Huneeus (I998), Correa, Figueroa, Jocelyn-Holt, Rolle y Vicuña (2002) y Marshall y Waissbluth (2007); así como en el trabajo de Rodríguez y Saavedra (2005). 
votos a la segunda mayoría; en caso contrario es elegido quien obtuvo más votos dentro de la coalición de la segunda mayoría. Este sistema de elección de legisladores no es proporcional y genera exclusión social al dejar fuera a grupos minoritarios, aunque se trate de grupos con alta votación aunque menor al 33\%. Es muy difícil que las mayorías elijan a los dos representantes, por lo cual casi siempre la segunda coalición puede imponer el veto a iniciativas legislativas del Poder Ejecutivo, pues la igualdad de representantes se asegura con sólo un tercio de las votaciones, o incluso menos si hay otras coaliciones que participan con una proporción alta de votos. En consecuencia, basta con tener un tercio de las preferencias populares para detener incluso modificaciones legales de mayoría simple. Ni hablar de aquellas que requieren quórum calificado. En tal sentido, y siguiendo a la literatura de ciencia política, se configura en Chile un caso preclaro de 'tiranía de las minorías'.

Ciertamente este modo de elegir legisladores no es casual. Responde a la estrategia de los creadores de la Constitución por evitar que los gobiernos democráticos contrarios al neoliberalismo la modificaran y, en su opinión, desnaturalizaran. Actualmente se han hecho más de cien enmiendas a la Constitución, muchas de ellas menores; pero a la fecha permanece inalterable el sistema electoral binominal.

Como contracara a su problema de representatividad, este sistema tiene las ventajas de facilitar la agrupación de partidos políticos en coaliciones y de otorgar estabilidad política al país. En cuanto al incentivo a agruparse, es claro que un partido de tamaño medio, que posea entre un Io\% y un $20 \%$ de apoyo político, no tiene opciones de elegir un representante si acude a la elección sin otro apoyo; sin embargo, es una muy buena opción y probablemente elija sus propios representantes si es que forma una coalición con otro $u$ otros partidos de igual o menor tamaño relativo (de $7 \%$ a $15 \%$ ). En consecuencia, la formación de dos grandes bloques en Chile no surge sólo por el amigo/enemigo común a quien defender/ atacar (Pinochet), sino que responde a la simple lógica de acceder al poder. En efecto, la Alianza agrupa a los dos principales partidos de derecha y la Concertación hizo lo propio con los principales cuatro partidos de centro e izquierda. Los extremos estaban fuera de ambas coaliciones. ${ }^{6}$

${ }^{6}$ Con la incorporación del Partido Comunista a la Nueva Mayoría (2013) se tiende a romper esta tendencia de dos grandes bloques de centro en el país. Sus consecuencias sobre los acentos en la equidad y la continuidad del modelo económico-político, en particular la estabilidad del sistema binominal, está aún por verse. 
También el sistema binominal entrega estabilidad política por cuanto exige que cualquier modificación legal requiera alcanzar acuerdos con sus oponentes. Eso es muy claro en las leyes que exigen quórum calificado, pero también lo es en leyes de quórum simple pues, como en toda coalición, el gobierno de turno carece del compromiso y honra de todos sus representantes para apoyar iniciativas legales. Cada legislador sabe que con este virtual empate su voto tiene un gran valor estratégico, debido a un poder monopólico inframarginal, lo que lleva a la aparición de senadores y diputados díscolos que negocian con el gobierno favores especiales para su distrito electoral. Como respuesta, muchas veces el gobierno llega a acuerdos con la oposición para ciertas modificaciones legales (típicamente de mercado), pues en general los díscolos están en los extremos del espectro político y son cuantitativamente menores. Patricio Aylwin llamó a esta estrategia de acuerdos la 'política de los consensos'.

Otras instituciones relevantes de mencionar en el ámbito político son la existencia de mecanismos de checks and balances a los poderes Ejecutivo y Legislativo, tal como la Contraloría General de la República y el Tribunal Constitucional. La Contraloría General de la República no es nueva; se trata de un órgano del Estado creado en I927 para controlar que los actos del gobierno se enmarquen en la legalidad vigente. Su cabeza máxima, el Contralor General de la República, es propuesto por el Presidente y ratificado por el Senado, con lo cual se propende hacia un ente independiente de ambos poderes del Estado.

El otro mecanismo de contrapeso es el Tribunal Constitucional creado en la Constitución de i980. Este tribunal se pronuncia por las decisiones de ambos poderes en cuanto a la legalidad constitucional de sus actos, por lo cual muchas veces es considerado una institución redundante. Asimismo, su existencia es bastante cuestionada por considerársele poco democrática, ya que sus miembros son nombrados por el Presidente previa ratificación del Senado y no por votación popular.

Con todo, ambas instituciones que hacen de contrapeso a las decisiones de los poderes ejecutivo y legislativo son un mecanismo adicional que entrega estabilidad y reduce los riesgos de comportamientos caprichosos por parte de quienes detentan el poder. 
Es importante mencionar, sin entrar en mucho detalle, que en los últimos quince años se han hecho dos reformas clave para fortalecer la institucionalidad política en Chile en el marco de la modernización del Estado. Luego de más de tres siglos de una manera arcaica de hacer justicia, se modificó el sistema procesal penal, cuyo código databa desde I874. Esta reforma se implementó gradualmente desde i998 hasta el año 2004. Se separó el rol de investigación, radicado ahora en las fiscalías, del rol de decisión, que permanece en los jueces; además de dar paso a juicios orales, más cortos y con salidas opcionales. Esta modernización de la justicia es un primer paso hacia el reto de garantizar a los chilenos y extranjeros con intereses en el país que sus derechos están cautelados y que la justicia opera en forma eficiente.

En paralelo, en 2003 se implementó el Sistema de Alta Dirección Pública. Este es un organismo que quitó el poder que tenía el Presidente de la República para nombrar a su arbitrio funcionarios públicos de rango medio, como superintendentes y fiscales de reparticiones públicas, lo que debería dar continuidad a la administración pública más allá de la rotación de los gobiernos y sus gobernantes.

En cuanto a la institucionalidad económica, una diferencia entre el gobierno militar y los gobiernos democráticos, que ayudó fuertemente a cambiar el modelo desde uno neoliberal a uno social de mercado, es la creación en los años I980 de instituciones necesarias para corregir las fallas del modelo heredado. Es así como se dota de independencia al Banco Central de Chile y se crean los marcos regulatorios de servicios básicos.

Respecto a lo primero, el diagnóstico de las causas de la debacle económica que culminó con una inflación desatada hacia fines del gobierno de Allende fue la dependencia que tenía el Banco Central del gobierno de turno. Esto llevó a que la irresponsabilidad fiscal se perpetuara en tanto todo déficit fiscal podía ser financiado con emisión de dinero. La independencia del Banco Central es, por tanto, una regla constitucional que corta de raíz el financiamiento fiscal por la vía del señoreaje y del impuesto de inflación.

Enla práctica esta independencia no es total, pues existen reuniones periódicas de coordinación entre el Banco Central y el Ministerio de Hacienda respecto de la política macroeconómica y crediticia en 
el país, además de ciertas prerrogativas del Ministro de Hacienda en cuanto a proponer acuerdos a tratar en el Consejo del Banco Central o suspender temporalmente la aplicación de acuerdos o resoluciones de este organismo. Sin duda, el Banco Central ha dado fuertes señales de independencia en ciertos momentos, cuando la inflación se ha elevado por sobre las metas esperadas. En esos episodios ha quedado claro que, a pesar de la preocupación por la producción y el empleo que tiene el Ejecutivo, el Banco Central demuestra que su objetivo inflacionario no sólo es el más importante sino el que se privilegia sobre los otros objetivos políticos.

El marco regulador de los servicios básicos en Chile data de 1982. Este fue el primer país que estableció los principios de la desregulación o liberalización de los mercados de energía eléctrica, telecomunicaciones, hidrocarburos y, posteriormente, de agua potable y alcantarillado. Inglaterra hizo lo propio, aunque aceleró la privatización de empresas en el año I984. Las primeras reformas fueron pensadas para establecer una estructura regulatoria (por mercados), lo que se hizo en Chile en I982 para prácticamente todos los sectores mencionados, salvo el sanitario que se hizo recién en I990. El marco regulador tarifario tomó más tiempo, quedando definido hacia fines de los años i980. En lo que respecta a hidrocarburos, estos quedaron liberalizados en i982, aunque en la práctica, y por razones de poder de mercado, la empresa estatal ENAP controla los precios de refinación aunque con criterios técnicos claramente definidos.

La elaboración de un marco regulador en estos sectores es tremendamente importante por dos razones económicas principales. En primer lugar, separa verticalmente cada una de estas industrias de forma tal que queda identificado cuál es el mercado monopólico necesario de regular y a cuál o cuáles mercados relacionados puede introducírsele competencia. En segundo lugar, entrega un método preciso de regulación tarifaria de forma que los precios regulados aseguren el autofinanciamiento de la empresa y, por otro lado, den señales de escasez e incentiven la inversión en estos sectores.

La competencia en los demás mercados no monopólicos se consideraba un hecho en el modelo neoliberal de los I980. La institucionalidad de promoción y defensa de la libre competencia era 
muy débil mientras que la de protección del consumidor simplemente no existía. Desde i973, Chile contaba con un sistema de dos comisiones antimonopolios ad hoc para casos de prácticas anticompetitivas: la preventiva y la resolutiva. La primera estaba compuesta por comisionados nombrados por diversos ministerios que sesionaban ad honorem medio día a la semana, mientras que los de la Comisión Resolutiva eran comisionados que operaban ad honorem, sesionaban una tarde a la semana y dos de ellos eran funcionarios del gobierno, otros dos sorteados de entre decanos de universidades (uno entre facultades de derecho y otro entre facultades de economía) y el quinto era un juez de la Corte Suprema. La gratuidad de su trabajo, la poca preparación con los casos, la nula independencia del gobierno y lo poco especializado de estos jueces ciertamente se confabulaban para que no se tomara con seriedad este sistema de protección de la libre competencia.

Fue así como en 1997, 2004 y 2009 se aprobaron tres leyes de gran importancia para el tema. En I997 se aprobó una ley que dio más estructura y recursos a la Fiscalía Nacional Económica, órgano investigador del Estado y facultado para hacerse parte en juicios de esta naturaleza y para actuar de oficio cuando el interés común estuviera en peligro. Una reforma mayor se produjo en 2004, cuando se eliminaron las comisiones antimonopolios y se les reemplazó por el Tribunal de Defensa de la Libre Competencia. Este tribunal entró en la pirámide judicial con rango de corte de apelaciones y cuatro de sus cinco miembros son funcionarios expertos (dos economistas y dos abogados) elegidos por cuatro años por sus propios méritos, cuya elección es nombrada por un comité de búsqueda liderado por el Banco Central que da credibilidad a la independencia de este tribunal. El quinto miembro es nombrado por la Corte Suprema. Una nueva enmienda a la Ley Defensa de la Libre Competencia fue aprobada en 2009 , la que dio aún más independencia al tribunal, elevó las multas máximas y entregó mayores atribuciones a la Fiscalía Nacional Económica, en particular para detectar y perseguir la formación de acuerdos colusivos.

La protección de los consumidores es otro aspecto totalmente descuidado de la agenda neoliberal de los años i980. El primer gobierno democrático tomó como uno de sus principios el buscar igualar las relaciones de poder entre empresas/comercio y los consumidores. 
Ya en 1982 se creó al alero del Ministerio de Economía el Servicio Nacional del Consumidor (SERNAC), entidad que proveía al público de información sobre ciertos mercados y orientaba a los consumidores en caso de que quisieran entablar una demanda. Sólo en el año I997 se aprobó la ley en que se formalizó su institucionalidad y se normó respecto de los procedimientos a seguir en juicios por abuso en contra de los consumidores. Si bien este fue un paso adelante, éste ha sido considerado muy tardío y con sabor a poco (Engel I998).

De cuatro modificaciones hechas a esta ley en sus quince años de vigencia, dos son interesantes de destacar. En 2004 el gobierno de Ricardo Lagos logró aprobar consensuadamente tres aspectos sustanciales respecto de los derechos de los consumidores. Primero, elevó las multas máximas en forma importante, aunque ciertamente irrisorias para las rentas que pueden dejar prácticas masivas de engaño a los usuarios. En segundo lugar, tipificó el delito de contrato de adhesión, lo que permite a los usuarios protegerse de cláusulas abusivas. Finalmente, y lo más importante, se logró aprobar una modificación que permite a las personas demandar colectivamente (class actions) a empresas que masivamente engañen a sus usuarios, en lo que la ley llamó protección de los derechos colectivos y difusos. En el año $201 \mathrm{II}$ se modificó nuevamente esta ley para normar de mejor manera temas relacionados al mercado financiero, tanto en el ámbito de la información disponible para los consumidores como en lo referido al rol del SERNAC en el incremento de la transparencia en dicho mercado.

Esta breve reseña nos muestra cómo se pasó de un modelo neoliberal fuerte en instituciones que protegían los derechos de propiedad y reducían la acción del Estado en los negocios privados, a otro en que las bases de la economía de mercado siguen intactas pero en donde se ha fortalecido la institucionalidad por la vía de generar contrapesos en la relación de la empresa con los usuarios.

El desarrollo de las instituciones en Chile puede ser puesto en perspectiva comparada utilizando lo que el Banco Mundial llama indicadores de Gobernabilidad (Kaufmann, Kraay y Mastruzzi 20I0).

En breve, estos indicadores capturan seis dimensiones que permiten juzgar la calidad institucional o gobernabilidad de un país para 2I5 países y territorios, estandarizándose los resultados de forma tal que 
el peor país en cada dimensión obtiene un -2,5 y el mejor país obtiene un $+2,5$, mientras que el país mediano obtiene cero. Los indicadores son Voz y Rendición de Cuentas (disponibilidad del gobierno para rendir cuentas, libertad de expresión, prensa competitiva), Estabilidad Política (percepción sobre posibles amenazas de violencia generadas con la intención de desestatibilizar o derrocar al gobierno), Efectividad Gubernamental (competencia de la burocracia, calidad de los servicios públicos y de la administración pública), Calidad Regulatoria (capacidad del gobierno para formular y ejecutar políticas acertadas que permitan y promuevan el desarrollo del sector privado), Certeza Jurídica (protección de derechos de propiedad y estado de derecho) y Control de la Corrupción (ausencia del abuso de la función pública para la ganancia privada).

El Gráfico 5 muestra que Chile tiene un desarrollo de su gobernabilidad consistente con los países de la OECD, y muy por encima del promedio de Latinoamérica. Entre los países emergentes, Chile se destaca por tener mejores resultados en general comparados a países industrializados como Italia, Grecia o Portugal, por ejemplo. En lo particular, al año 20I2 Chile tenía mejores indicadores de gobernabilidad que los demás países de América Latina, salvo en estabilidad política en donde en términos relativos Chile ha visto reducido su indicador al cuarto lugar de entre los países de la región.

- GRÁFICO 5. INDICADORES DE GOBERNABILIDAD EN 2012

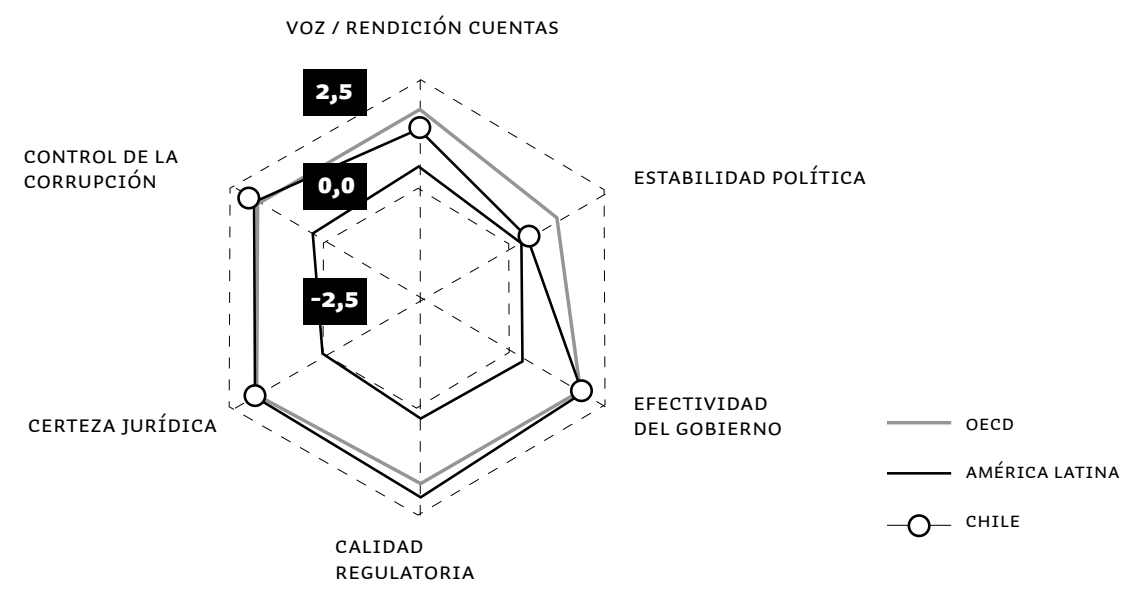


De acuerdo al mismo Banco Mundial, en los últimos ıo años Chile ha avanzado o mantenido un alto estándar en estos índices de gobernabilidad, salvo nuevamente en estabilidad política. La razón de esta baja en el índice de estabilidad política podría deberse a que se trata de un índice relativo, por lo que reflejaría una mayor estabilidad política en otros países en vías de desarrollo; pero también podría deberse a que esta caída sería un indicador de la baja cohesión social en Chile que ha llevado a cuestionamientos abiertos al modelo de desarrollo económicopolítico imperante en el país (ver el Gráfico 6).

- GRÁFICO 6. EVOLUCIÓN DE LOS INDICADORES DE GOBERNABILIDAD

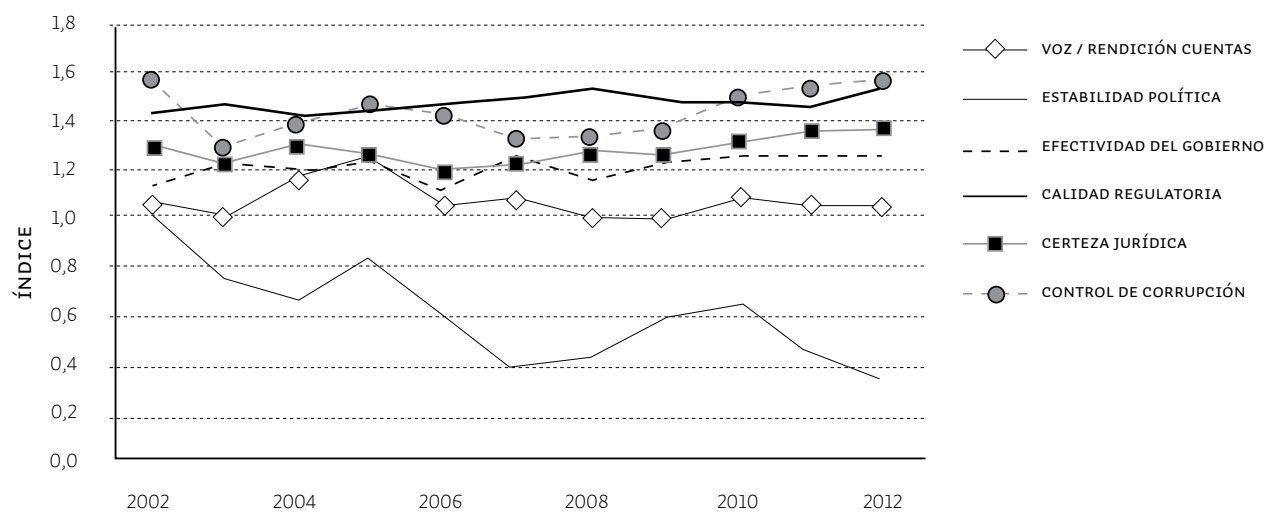

FUENTE: ELABORACIÓN PROPIA BASADO EN WORLD BANK 2014

\section{Consecuencias institucionales en el clima de negocios}

De la discusión previa es posible deducir que la economía chilena se caracteriza por contar con reglas de juego claras, cuya certeza de cumplimiento y de eficacia de los gobiernos generan confianzas en los inversionistas. Todo ello ha rendido frutos en términos de un sostenido crecimiento económico.

La ecuación que muestra esta relación causal es sencilla: las reformas liberales del gobierno militar en los años I970 y I980, unido a la profundización de políticas pro-competitivas (junto a una política 
social activa) llevadas a cabo por los sucesivos gobiernos democráticos, entregan buenas señales para un mejor clima de negocios. Esta simple relación causal tiene asidero teórico y empírico más fuerte en la literatura especializada, como los trabajos de North (I990), quien plantea que la fortaleza de las instituciones es una precondición al crecimiento económico, o de Parente y Prescott (2000), quienes muestran que los países con mayor ingreso per cápita protegen de mejor manera la libre competencia.

Una muestra de ese buen clima de negocios en Chile es la evolución de la inversión, particularmente aquella proveniente del extranjero. Durante todo el gobierno militar la inversión extranjera materializada desde I974 a I989 alcanzó los Iı.800 millones de dólares (en moneda del año 2000); mientras que subió a poco más de 75 mil millones de dólares desde I990 a 2006, igual monto que se cubrió sólo entre 2007 y 20II. Más aún, sólo en 2012 esta cifra alcanzó los treinta mil millones de dólares. Esto es, anualmente la inversión extranjera desde la vuelta a la democracia ha sido quince veces más alta que aquella prevaleciente en el gobierno militar. Sin duda, esta información es una muestra de que con los mismos instrumentos la certeza que da a los inversionistas una economía de mercado en democracia es muy diferente al de una economía de mercado en dictadura.

Otra muestra de este clima de negocios es la percepción que se tiene de la calidad de las instituciones y lo atractivo de hacer negocios en Chile. Sólo a modo de ejemplo, el índice de ambiente de los negocios de The Economist Intelligence Unit muestra a Chile regularmente por debajo del puesto 20 a nivel global, de un total de 82 países, superando a países como Israel, Malasia e incluso Corea del Sur. De acuerdo a este ranking, en abril de 20ı Chile ocupaba el puesto I4 para el ranking de los años 2014 a 20I8. Siempre, y en forma consistente, ha sido el número uno de América Latina para dicho ranking. ${ }^{7}$

Otra medida que permite visualizar cómo en rasgos generales Chile ha tenido un buen desempeño al momento de favorecer el clima de negocios, es el tiempo que toma crear una empresa. En Chile se requieren siete trámites para hacer esta tarea, dos menos que el promedio de América Latina, pero dos más que en los países de la OECD. Sin

${ }^{7}$ Detalles pueden ser consultados en www.eiu.com. 
embargo, en términos de duración de esos trámites, Chile con 5,5 días está en la mitad de lo que toma en los países más desarrollados (II,I días) y ciertamente mucho mejor que en el resto de la región (36,I días). A nivel global, la posición de Chile respecto de la apertura de nuevos negocios es de privilegio, estando en el año 2013 en el puesto 22 de un total de I82 países considerados. En términos de su evolución, en este mismo ranking Chile se movía entre los puestos 40 y 55 hasta hace unos cinco años atrás, lo que muestra el avance en esta materia en el último quinquenio. $^{8}$

\section{Conclusiones}

Chile muestra altas fortalezas institucionales en lo económico y político, las que, unidas, han sido capaces de crear las condiciones para el crecimiento sostenido y la consecuente reducción de la pobreza, que ha bajado desde un 38,4\% el año I990 a I4.4\% en el año 20II. En otras palabras, se puede decir que las bases del modelo chileno son sólidas en sus variables fundamentales; incluso podría decirse que el pecado original de este modelo ha sido 'limpiado' con una buena crianza.

Algunas reformas en la institucionalidad económica y en la política aún son requeridas. Por de pronto, en las primeras se requiere avanzar en la independencia, separación de tareas y fortalecimiento de reguladores en energía, telecomunicaciones, bancos e instituciones financieras e infraestructura; crear un tribunal económico que vea causas comerciales y englobe además las tributarias, las de consumidores y las de libre competencia; avanzar hacia una estructura de gobierno clara y eventual privatización de las principales empresas públicas: CODELCO y ENAP; definir y repensar el rol empresarial del Estado en áreas sensibles, como la de pensiones y seguros de salud, por ejemplo; y dotar sin complejos a las instituciones fiscalizadoras del Estado los recursos económicos, técnicos y legales necesarios para ejercer su tarea con eficacia, en particular la tarea de defensa de los derechos de los consumidores que recae en el SERNAC.

\footnotetext{
${ }^{8}$ Detalles pueden ser consultados en http://espanol.doingbusiness.org. Es importante destacar que la promulgación de la Ley 20.494 en el año 20II, durante el gobierno de Sebastián Piñera, eliminó un trámite y agilizó varios más, lo que llevó a reducir en I/4 los tiempos de aperturas de nuevos negocios en el país.
} 
En materia de reformas políticas está pendiente avanzar más aún en la profesionalización de empleados y directivos públicos, en la regulación del financiamiento de las actividades políticas, y en los mecanismos para dotar a los ciudadanos el derecho a ser escuchados y elegir sus representantes con un mayor grado de proporcionalidad que el actualmente vigente.

De no avanzar más en estas modificaciones mencionadas, la exclusión social y política que le subyace al modelo económico-político chileno incuba su propio fin. En particular, el gran desafío se encuentra en reducir o eliminar la exclusión de grupos minoritarios pues la desigualdad (de ingresos pero más aún de oportunidades), la exclusión social subsecuente y la exclusión política son alarmantes y comprometen el progreso económico y social de Chile. Luego el gran dilema económico político sigue siendo ¿cómo lograr una mayor cohesión social en el país?

Ciertamente, el que los sucesivos gobiernos de la Concertación (I990-20I0) y el de la Alianza (20I0-20I4) poco hicieran respecto de la cohesión social, llevó a muchos sectores a desconfiar del 'modelo económico' al elegir a Michelle Bachelet para un nuevo gobierno entre 2014 y 2018 , con promesas concretas hacia una mayor equidad. Reformas profundas en materia laboral, previsional, energética y sobre todo educacional derechamente apuntan a un modelo de corte desarrollista, alejado del de libre mercado con progreso social que Chile ha experimentado en los últimos veinte años (Riesco 2009).

En suma, poner el objetivo de equidad por sobre el de crecimiento económico, y con ello por sobre el de pobreza, nos plantea la pregunta de si estamos de vuelta o a la búsqueda de un modelo alternativo. 


\section{BIBLIOGRAFÍA}

Acemoglu, D. y Robinson, J. 2012. ¿Por qué fracasan los países? Los orígenes del poder, la prosperidad y la pobreza. Madrid: Deusto Ediciones.

Bizberg, I. 20I2. Types of Capitalism in Latin America. Revue Interventions Économiques 47. [Online] Disponible en: http://crbc.ehess.fr/docannexe/ file/20I4/24_mars_2oI4_brics_interventionseconomiques_bizberg.pdf [20 de marzo 20I4].

Bourguignon, F. 2004. The Poverty-Growth-Inequality Triangle. Mimeo The World Bank. [Online] Disponible en: http://siteresources.worldbank.org/ INTPGI/Resources/342674-I206III890I5I/I5I85_ICRIER_paper-final. $\operatorname{pdf}[20$ de marzo 20I4].

CASEN varios años. Encuesta CASEN. Ministerio de Desarrollo Social. [Online] Disponible en: http://observatorio.ministeriodesarrollosocial.gob.cl/ casen_obj.php [20 de marzo 20I4].

CEPAL 20I4. Base de datos y publicaciones estadísticas. [Online] Disponible en: http://estadisticas.cepal.org/cepalstat/WEB_CEPALSTAT/Portada.asp [20 de marzo 20I4].

Correa, S., Figueroa, C., Jocelyn-Holt, A., Rolle, C., y Vicuña, M. 200I. Documentos del Siglo XX Chileno. Santiago: Editorial Sudamericana.

Edwards, S. 200I. Veinticinco años de inflación y estabilización en Chile (1973I998). En Larraín, F. y Vergara, R. (eds), La transformación económica de Chile. Santiago: Centro de Estudios Públicos.

Engel, E. I998. Protección de los consumidores en Chile: ¿Por qué tan poco y tan tarde? Perspectivas en Política, Economía y Gestión I(2), I45-I66.

Ffrench-Davis, R. 2001. Entre el neoliberalismo y el crecimiento con equidad. Santiago: Dolmen.

Foxley, A. I993. Economía política de la transición. Santiago: Dolmen.

Foxley, A. 2005. Lessons from Chile's Development in the 9os. En Besley, T. y Zagha, R. (eds), Development Challenges in the 1990s. Leading Policymakers Speak from Experience. Washington DC: The World Bank.

Fuentes, R. 20I3. Evidencia para Chile. En Acuña, R. (coord), Contribución del sistema privado de pensiones al desarrollo económico de Latinoamérica: Experiencias de Colombia, México, Chile y Perú. Estudio realizado por SURA Asset 
ManagementASSET. [Online] Disponible en: http://www.fiap.cl/prontus_ fiap/site/artic/20ıзгол/asocfile/2013гогог8ıiз/librointeractivo.pdf [20 de marzo 20I4].

Huneeus, C. I998. Chile's New Democracy: Political Funding and Economic Transformation. En Burnell, P. y Ware, A. (eds), Funding Democratization. Manchester: Manchester University Press.

Kaufmann, D., Kraay, A., y Mastruzzi, M. 20ıо. The Worldwide Governance Indicators: Methodology and Analytical Issues. World Bank Policy Research WPS 5430. [Online] Disponible en: http://www.brookings.edu/ /media/ research/files/reports/2010/9/wgi\%2okaufmann/o9_wgi_kaufmann [20 de marzo 20I4].

Keefer, P. 2004. What Does Political Economy Tell Us About Economic Development -and ViceVersa? World Bank Policy Research WPS 3250. [Online] Disponible en: http://elibrary.worldbank.org/doi/ pdf/IO.I596/18I3-9450-3250 [20 de marzo 20I4].

Landerretche, O. 2005. Construyendo solvencia fiscal: El éxito macroecónomico de la Concertación. En Meller, P. (ed), La paradoja aparente. Equidad Y eficiencia: Resolviendo el dilema. Santiago: Ediciones Taurus.

Larraín, F. y Vergara, R. 2ooı. Chile en pos del desarrollo: Veinticinco años de transformaciones económicas. En Larraín, F. y Vergara, R. (eds), La transformación económica de Chile. Santiago: Centro de Estudios Públicos.

Marshall, J. y Waissbluth, M. 2007. Reforma del Estado en Chile: Una oportunidad. En Varios Autores, Institucionalidad para el desarrollo: Los nuevos desafíos. Santiago: Expansiva y Centro de Estudios Públicos.

Menard, C. 2006. Hybrid Organization of Production and Distribution. Revista de Análisis Económico 2I(2), 25-4I.

Meller, P. 2005. Una Revisión del debate ideológico-económico sobre equidad y crecimiento. En Meller, P. (ed), La paradoja aparente. Equidad y eficiencia: Resolviendo el dilema. Santiago: Ediciones Taurus.

North, D. I990. Institutions, Institutional Change, and Economic Performance. Cambridge: Cambridge University Press.

Parente, S. y Prescott, E. 2000. Barriers to Riches. Cambridge, Mass.: The MIT Press.

Raczynski, D. y Serrano, C. 2005. Las políticas y estrategias de desarrollo social. Aportes de los años gos y desafíos futuros. En Meller, P. (ed), La paradoja aparente. Equidad y eficiencia: Resolviendo el dilema. Santiago: Ediciones Taurus. 
Riesco, M. 2009. El modelo social chileno comienza a cambiar. Revista Internacional del Trabajo I28(3), 3II-330.

Rodríguez Grossi, J. y Saavedra, E. 2005. Certeza jurídica e incentivos a la inversión: Política y práctica de una relación causal. Persona y Sociedad I9(2), 55-75.

Rodríguez Grossi, J. y Saavedra, E. 2008. Chile. En Fischer-Bollin, P. y Saavedra, E. (eds), Crecimiento y progreso social en América Latina. São Paulo: Konrad Adenauer Stiftung.

Schneider, B. y Soskice, D. 2009. Inequality in Developed Countries and Latin America: Coordinated, Liberal and Hierarchical Systems. Economy and Society 38(I), I7-52.

Recibido diciembre 2013

Aceptado marzo 2014

Clasificación JEL: Oı2, O54, Pi6 